\title{
DAVIS-WIELANDT SHELLS OF OPERATORS
}

\author{
ChI-Kwong Li, Yiu-Tung PoOn And Nung-Sing Sze
}

Abstract. Basic properties of Davis-Wielandt shells are presented. Conditions on two operators $A$ and $B$ with the same Davis-Wielandt shells are analyzed. Special attention is given to the case when $B$ is a compression of $A$, and when $B=A^{*}, A^{t}$, or $\left(A^{*}\right)^{t}$, where $A^{t}$ is the transpose of $A$ with respect to an orthonormal basis. The results are used to study the point spectrum, approximate point spectrum, and residual spectrum of the sum of two operators. Relation between the geometrical properties of the Davis-Wielandt shells and algebraic properties of operators are obtained. Complete descriptions of the Davis-Wielandt shells are given for several classes of operators.

Mathematics subject classification (2000): 47A10, 47A12, 47B15.

Key words and phrases: Davis-Wielandt shells, generalized numerical ranges, spectra, operators.

\section{REFERENCES}

[1] J. B. Conway, A Course in Functional Analysis, Second edition, Graduate Texts in Mathematics, 96, Spring-Verlag, New York, 1990.

[2] C. DAVIS, The shell of a Hilbert-space operator, Acta Sci. Math. (Szeged) 29 (1968), 69-86.

[3] C. DAVIS, The shell of a Hilbert-space operator. II, Acta Sci. Math. (Szeged) 31 (1970), 301-318.

[4] K. E. Gustafson AND D. K. M. RAO, Numerical ranges: The field of values of linear operators and matrices, Springer, New York, 1997.

[5] P. Halmos, A Hilbert Space Problem Book, Second edition, Graduate Texts in Mathematics, 19, Encyclopedia of Mathematics and its Applications, Spring-Verlag, New York, 1982.

[6] R. A. Horn And C. R. Johnson, Topics in Matrix Analysis, Cambridge University Press, Cambridge, 1991.

[7] C. K. Li And Y. T. PoOn, Convexity of the joint numerical range, SIAM J. Matrix Analysis Appl. 21 (1999), 668-678.

[8] C. K. LI, Y. T POON AND N. S. SzE, Eigenvalues of the sum of matrices from unitary similarity orbits, SIAM J. Matrix Analysis Appl., to appear.

[9] H. WiElandT, On eigenvalues of sums of normal matrices, Pacific J. Math. 5 (1955), 633-638. 